\title{
Mathematical modeling of the effects of public health education on tungiasis- a neglected disease with many challenges in endemic communities
}

\author{
Rachel A. Nyang'inja ${ }^{1,2 *}$, David N. Angwenyi ${ }^{3}$, Cecilia M. Musyoka' and Titus O. Orwa ${ }^{4}$
}

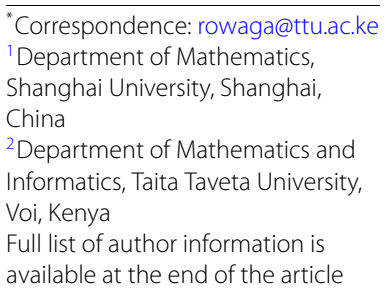

\begin{abstract}
In this paper, we formulate and study a mathematical model for the dynamics of jigger infestation incorporating public health education using systems of ordinary differential equations and computational simulations. The basic reproduction number $R_{E}$ is obtained and used to determine whether the disease breaks out in the population and results in an endemic equilibrium or dies out eventually corresponding to a disease-free equilibrium. We carried out an analysis of the model and established the conditions for the local and global stabilities of the disease-free and endemic equilibria points. Using the Lyapunov stability theory and LaSalle invariant principle, we found out that the disease-endemic equilibrium point is globally asymptotically stable if $R_{E}>1$ and unstable otherwise. Numerical simulations are performed to illustrate our theoretical predictions. Both the analytical and numerical results show public health education is a very effective control measure in eradicating jigger infestation in the endemic communities at large.
\end{abstract}

Keywords: Basic reproduction number; Global stability; Jigger infestation; Lyapunov stability theory; Public health education

\section{Introduction}

Tungiasis is a parasitic skin disease caused by female sand flea or jigger (tunga penetrans) that mostly lives in dry sand and soil [1]. The impregnated female parasite attacks exposed skin and burrows itself into the skin, leaving a section of its abdomen exposed through a pore in the skin to enable it breathe and carry out other metabolic functions like feeding on blood vessels [2]. The first evidence of jigger infestation is a tiny dark spot on the skin at the point of penetration. When the eggs are ready, they are released through the orifice and after about one week hatch into fleas [3]. It is important to note that one female flea may lay hundreds of eggs, meaning that if the situation is not promptly corrected it can easily result into severe health effects.

Tungiasis is endemic in many countries including Latin America, the Caribbean, and Sub-Sahara Africa [4]. Researchers agree that it is a neglected health problem in impoverished communities in the affected countries [5]. In Kenya, the neglect is evidenced by scarcity of epidemiological data [6]. It is estimated that about 2.6 million (6.5\%) Kenyans

(c) The Author(s) 2018. This article is distributed under the terms of the Creative Commons Attribution 4.0 International License (http://creativecommons.org/licenses/by/4.0/), which permits unrestricted use, distribution, and reproduction in any medium, provided you give appropriate credit to the original author(s) and the source, provide a link to the Creative Commons license, and indicate if changes were made. 
suffer from tungiasis and 10 million others are at risk [7]. Tungiasis is a common problem among people who live in Mount Kenya region, western Kenya, Rift Valley, and coastal regions [2]. In 2010, the prevalence of tungiasis in Muranga South, an endemic area, was $57 \%$ among children aged 5 to 12 years [8]. Unhygienic conditions have been identified as the major causes of tungiasis [9]. The jigger menace is a common problem among the low income populations, particularly where people live with furred animals under congested and filthy conditions [10]. Dirty and warm areas are optimal breeding grounds for the flea. Severe tungiasis infestations occur in vulnerable age groups such as children and the elderly, especially those from the economically challenged communities [11]. Jiggers cause extreme local itching, pain, and sensation in the skin of their victims. Other effects include: restlessness, edginess, poor appetite, susceptibility to opportunistic infections, lymphangitis, gangrene and extreme limb amputations, sepsis, loss of toes, poor health, poor school performance and drop out, low self-esteem, permanent distortion of shape of legs and hands, and can be life threatening [12].

Treatment of tungiasis involves identification of the parasite especially through mechanical removal using a sterile, sharp pointed object such as a needle or pin followed by an antiseptic dressing. It may also be effectively treated by surgical extraction of embedded sand fleas under sterile conditions in medical facilities [13]. The fleas may also be deterred by washing the affected areas with disinfectants like potassium permanganate, topographic application of anti-parasitic agents, use of anti-inflammatory creams and use of repellents [14]. Personal hygiene and wearing of shoes are key in controlling jigger infection. Children and the elderly need to be well taken care of through provision of primary and affordable amenities and healthcare facilities within close proximity of low income populations.

The transmission of the infectious diseases has been of great interest to both medics and scholars [15]. Public health programs play a very important role in defining health issues since they serve as a major source of information and influence changes in the way people behave. It is, therefore, critical to study epidemic transmission and take effective strategies to prevent, control, and contain them. Individuals response to a disease threat depends on risk perception that is gained largely through information reported by the government to the public, for example, a number of infections, hospitalizations, and deaths, as provided by the public health department [16, 17]. For instance, public health officials could be interested in epidemic measurements such as the peak number of infections, peak time, the total number of infections, and the end of epidemic, which are all directly related to important public health resources [18]. Public health programs, such as public vaccination or immunization, isolation, quarantine, and awareness creation through media, can affect disease transmission during an epidemic contagion [19].

Mathematical models can serve as powerful tools for understanding the driving mechanisms of disease transmission. A general susceptible-infected-recovered or removed model was studied in [20] by using type 2 functional response to depict the transmission rate decreased by awareness intervention. The authors established that effective awareness measures can reduce the number of infections. An effective method of reducing the spread of the disease is to make people understand the preventive measures as soon as possible. A mathematical model was presented in [21] to study the effects of public health educational campaigns as a single control strategy on sexual transmission of HIV/AIDS in the continuing absence of a preventative vaccine. Results showed that public health educational campaigns can slow down the epidemic. In [22], the authors formulated a 
deterministic model on HIV/AIDS transmission and studied the impact of educational programs and abstinence in Sub-Saharan Africa.

Despite the harm inflicted by tungiasis and countless challenges of the disease to the population, studies on the disease from mathematical modeling perspective are very scarce. Theoretical studies so far conducted on tungiasis have focused on the knowledge pertaining to the biology, epidemiology, and pathology of the jigger, possible causes of the disease, attitude and practices on the prevalence in different endemic communities [11, 12, 23]. Only a few works do exist in the literature that address the problem from a mathematical approach. Some of the challenges encountered in modeling of tungiasis disease and its control interventions include the limited understanding of how the disease manifests in humans, its transmission dynamics, the jigger flea, and the limited data on the disease that exist as most infested people shy away from seeking medical attention due to stigma that is associated with the disease. Recently, the authors in [24] spear-headed the application of mathematical models to tungiasis transmission dynamics. They formulated and studied a mathematical model for the dynamics of tungiasis transmission in zoonotic areas by conducting a sensitivity analysis to determine the parameters with high impact on the basic reproduction number which could be targeted with the control interventions. Their results showed that reducing the on and off-host flea population and the effective contact rate between the sand flea sources and the hosts would be effective intervention strategies to decrease the probability of a large transient of tungiasis epidemic. This modeling work was extended in $[25,26]$ to include stability and optimal control strategies with an aim to minimize on the number of infested humans, infested animals, and sand flea populations. In [27], the author employed a susceptible-infected-removed (SIR) model to study the dynamics of jigger infestation incorporating treatment as a control strategy against infestation. It was established that a high probability of success of treatment leads to a low jigger prevalence in a population. All of these modeling efforts have not only provided useful information for understanding the mechanics of tungiasis disease transmission, but also highlighted the need for better data to construct and validate future models of tungiasis. Disease spread is an important phenomenon that has been widely studied both in the fields of classical models [28-30] and in networks [31,32]. However, there are currently no models that comprehensively study the dynamics of tungiasis transmission with public health education as a control strategy, and that is our motivation for this study.

In this work, therefore, we theoretically study the dynamics of jigger infestation incorporating public health education using systems of ordinary differential equations and numerical simulations. Specifically, we formulate the epidemic threshold to enable us determine whether the infection breaks out in the population and results in an endemic equilibrium or dies out eventually corresponding to a disease-free equilibrium and predict the disease prevalence. We have comprehensively analyzed the model and proved that a unique positive endemic equilibrium exists and is locally and globally asymptotically stable. Besides, we have carried out numerical simulations to verify the results and demonstrate the validity of the various assumptions made during the analysis. The paper is organized as follows: in this section, we have provided background information of jigger infestation and given a brief discussion on previous works on the disease and other findings on a general impact of public health intervention measures on epidemics; in Sect. 2, we have formulated and described the mathematical model, presented its basic properties (positivity and boundedness), and determined its equilibrium points, the basic reproduction number $R_{E}$, and 
the existence and uniqueness of the disease positive endemic equilibrium. We have also established the sufficient conditions for the local and global stabilities of the disease-free and the disease-endemic equilibria and proved that they are both globally asymptotically stable. Section 3 presents numerical simulations to verify our theoretical results. Finally, we discuss and conclude this work in Sects. 4 and 5, respectively.

\section{The model}

The total human population size $N$ at time $t$ is subdivided into compartments: susceptible compartment $S(t)$, in which all individuals are susceptible to jigger infestation; educated compartment $E(t)$, in which all individuals are educated on jigger infestation prevention strategies or treatment, and infected compartment $I(t)$, in which all individuals are infested by the disease and have infectivity. Since the incubation period is very short, we assume that the probability of survival till the infectious state for the individuals exposed to jiggers is unity and exclude the exposure stage. At each time step, each individual exists in only one of the three stated compartments. Recruitment into susceptible population takes place at the rate $\Lambda$. We assume that the mass-action incidence transmission is defined by $\beta S I$, where $\beta$ is the effective contact rate for disease transmission. When the jigger infected individuals receive treatment or other interventions, they will be cured and become susceptible again with the recovery rate $\gamma$. The rate at which public health educational strategies disseminate among susceptible individuals as per the public health intervention programs is represented by $\varepsilon$. Since the influence of education may not be permanent or the strategies employed may not be very effective, the knowledge of taking control measures gradually wears off, and so educated individuals will be infected at a lower rate $\alpha \beta E I$, where $0<\alpha<1$. The fraction $\alpha$ reflects the effect of reducing the infection due to public health education. In this work, $0<\alpha<1$ because $\alpha=0$ means education is completely effective in preventing jigger infection, and $\alpha=1$ implies education is not useful at all. Disease mortality is assumed to take place at the rate $\sigma$, while $\mu$ is the natural death rate.

From the flowchart described in Fig. 1, we have the following SEI model with nonnegative initial conditions:

$$
\left.\begin{array}{rl}
\frac{\mathrm{d} S}{\mathrm{~d} t} & =\Lambda-\beta S I+\gamma I-(\varepsilon+\mu) S \\
\frac{\mathrm{d} E}{\mathrm{~d} t} & =\varepsilon S-\alpha \beta E I-\mu E \\
\frac{\mathrm{d} I}{\mathrm{~d} t} & =\beta S I+\alpha \beta E I-(\gamma+\sigma+\mu) I .
\end{array}\right\}
$$

\subsection{Positivity and boundedness of solutions}

We sum up the three equations in model system (1) and obtain the total population size as

$$
N(t)=S(t)+E(t)+I(t) .
$$




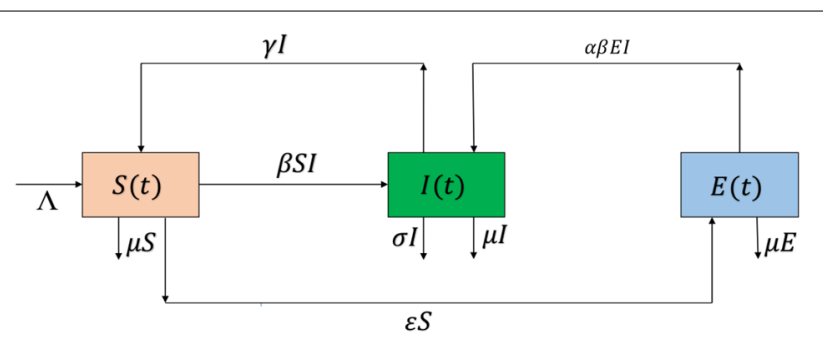

Figure 1 Flowchart of an SEl model with education. Humans are recruited into the susceptible class at a rate $\Lambda$. Susceptible individuals are infected by the infected persons at a per capita rate $\beta \mathrm{S} /$ and are educated at a per capita rate $\varepsilon$. Infected individuals recover to be susceptible again at a per capita rate $\gamma$. The educated individuals are infected at a reduced per capita rate $\alpha \beta E /$ due to ineffective educational intervention strategies. Per capita natural death rate and death rate due to jigger infestation are represented by $\mu$ and $\sigma$, respectively

This gives

$$
\begin{aligned}
& \frac{d N}{d t}=\frac{d S}{d t}+\frac{d E}{d t}+\frac{d I}{d t}, \\
& \frac{d N}{d t}=\Lambda-\mu N-\sigma I .
\end{aligned}
$$

Model system (1) describes human population, therefore all the associated state variables are nonnegative for all time $t>0$. The solutions of this model with positive initial data remain positive for all time $t>0$. The positive invariant region can be obtained using Theorem 1.

Theorem 1 The solutions of model system (1) are feasible for $t>0$ if they enter the invariant region $\Gamma$.

Proof Let $\Gamma=(S, E, I) \in \mathfrak{R}_{+}^{3}$ be any solution of the model equations with nonzero initial conditions. In the absence of infection or disease, equation (3) becomes

$$
\begin{aligned}
& \frac{d N}{d t} \leq \Lambda-\mu N, \\
& \frac{d N}{d t}+\mu N \leq \Lambda .
\end{aligned}
$$

The integrating factor for equation (5) is $e^{\mu t}$. Multiplying both sides of equation (5) by $e^{\mu t}$ gives

$$
\begin{aligned}
& e^{\mu t} \frac{d N}{d t}+\mu N e^{\mu t} \leq \Lambda e^{\mu t}, \\
& d\left[N e^{\mu t}\right] \leq \Lambda e^{\mu t} d t .
\end{aligned}
$$

Integrating both sides, we have

$$
\begin{aligned}
& d[t] e^{\mu t}=\frac{\Lambda e^{\mu t}}{\mu}+C, \\
& N(t)=\frac{\Lambda}{\mu}+C e^{-\mu t} .
\end{aligned}
$$


Applying the initial conditions $t=0, N(0)=N_{0}, N_{0} \leq \frac{\Lambda}{\mu}+C$, which implies

$$
\begin{aligned}
& N_{0}-\frac{\Lambda}{\mu} \leq C \\
& \Rightarrow \quad N \leq \frac{\Lambda}{\mu}+\left[N_{0}-\frac{\Lambda}{\mu}\right] e^{-\mu t} .
\end{aligned}
$$

Therefore, as $t \rightarrow \infty$ in equation (11), the human population $N$ approaches $L=\frac{\Lambda}{\mu}$ (i.e., $N \rightarrow L=\frac{\Lambda}{\mu}$ ). The parameter $L=\frac{\Lambda}{\mu}$ is called the carrying capacity.

Hence, $N$ is bounded and all feasible solution sets of the human population of model system (1) approach, enter, or stay in the region $\Gamma=\left\{(S, E, I) \in \mathbb{R}_{+}^{3}: S>0, I \geq 0, E \geq 0\right.$, $\left.N \leq \frac{\Lambda}{\mu}\right\}$.

Therefore, the region $\Gamma$ is positively invariant, that is, the solution is positive for all times $(t)$ and model system (1) is epidemiologically meaningful and mathematically well-posed in the domain $\Gamma$. Hence, it is sufficient to consider the dynamics of the flow it generates in a proper subset $\Gamma=\left\{(S, E, I) \in \mathbb{R}_{+}^{3}: N \leq \frac{\Lambda}{\mu}\right.$.

Theorem 2 All feasible solutions of model system (1) are uniformly bounded in the subset region $\Gamma$.

Proof Let the initial data set be $\{S(0)>0,[I(0), E(0) \geq 0]\} \in \Gamma$. Then the solution set $\{(S, E, I)\}(t)$ of model system (1) is nonnegative for all $t>0$.

Consider the first equation of model system (1) at time $t$.

Let $k=\beta I$, that is, the force of infection. Then

$$
\begin{aligned}
\frac{d S}{d t} & =\Lambda-k S-(\varepsilon+\mu) S+\gamma I \\
& =\Lambda-(k+\varepsilon+\mu) S+\gamma I .
\end{aligned}
$$

Now,

$$
\frac{d S}{d t} \geq-(k+\varepsilon+\mu) S .
$$

Separating variables in equation (12) and integrating both sides, we have

$$
\begin{aligned}
& \frac{d S}{S} \geq-(k+\varepsilon+\mu) d t, \\
& \int \frac{d S}{S} \geq-\int(k+\varepsilon+\mu) d t, \\
& \ln S \geq-(k+\varepsilon+\mu) t+C, \\
& S(t)=e^{-(k+\varepsilon+\mu) t}, \\
& S(t) \geq L e^{-(k+\varepsilon+\mu) t}, \quad \text { where } L=e^{C} .
\end{aligned}
$$

Using the initial conditions, $t=0$ implies $(0) \geq L$.

Therefore,

$$
S(t) \geq S(0) e^{-(k+\varepsilon+\mu) t} \geq 0 .
$$


From the third equation of model system (1), we have

$$
\frac{d I}{d t}=\beta S I+\alpha \beta E I-(\gamma+\sigma+\mu) I .
$$

Again, making the substitution $k=\beta I$, we have

$$
\frac{d I}{d t}=k S+\alpha k E-(\gamma+\sigma+\mu) I
$$

Then

$$
\frac{d I}{d t} \geq-(\gamma+\sigma+\mu) I
$$

Separating variables in equation (13) and integrating both sides gives

$$
\begin{aligned}
& \frac{d I}{I} \geq-(\gamma+\sigma+\mu) d t, \\
& \int \frac{d I}{I} \geq-\int(\gamma+\sigma+\mu) d t, \\
& \ln I \geq-(\gamma+\sigma+\mu) t+C, \\
& I(t)=k e^{-(\gamma+\sigma+\mu) t} .
\end{aligned}
$$

Applying the initial condition $t=0$ implies $I(0) \geq k$.

Therefore,

$$
I(t) \geq I(0) e^{-(\gamma+\sigma+\mu) t} .
$$

Similarly, it can be verified that the second equation of model system (1) is also positive for all time $t>0$, since $e^{\tau}>0$ for all $\tau \in \mathbb{R}$.

\subsection{Equilibrium points of the model}

In this section, we analyze the model to investigate the stability of its equilibria both at disease-free equilibrium (DFE) and at disease-endemic equilibrium (EE). The disease-free equilibrium points of the model are its steady state solutions in the absence of infection or disease [33]. To obtain the equilibrium points of model system (1), we equate the derivatives to zero and solve for the variables:

$$
\left.\begin{array}{r}
\Lambda-\beta S I+\gamma I-(\varepsilon+\mu) S=0 \\
\varepsilon S-\alpha \beta E I-\mu E=0 \\
\beta S I+\alpha \beta E I-(\gamma+\sigma+\mu) I=0 .
\end{array}\right\}
$$

Using $S+E=\frac{\Lambda}{\mu}$, it can be shown that the disease-free equilibrium of model system (1) is defined by

$$
D^{0}=\left(S^{0}, E^{0}, I^{0}\right)=\left\{\frac{\Lambda}{(\varepsilon+\mu)}, \frac{\Lambda \varepsilon}{\mu(\varepsilon+\mu)}, 0\right\} .
$$




\subsection{The basic reproduction number $R_{E}$}

The basic reproduction number $R_{E}$ is an important epidemiological parameter which is defined as the expected number of secondary infections produced by a single infective individual in a completely susceptible population [28]. Specifically, we define the basic reproduction number $R_{E}$ of model system (1) as the number of secondary jigger infections caused by a single jigger infected individual in the presence of public health educational interventions. When no such programs are employed, the basic reproduction number is defined by $R_{0}$. It measures the power of a disease to invade a population under conditions that facilitate maximal growth. In general, the basic reproduction number $R_{0}$ depends on the demographic, disease, and mobility parameters.

According to Van den Driessche and Watmough [34], and using the next-generation operator approach [28], the basic reproduction number can be computed by $R_{0}=\rho\left(F V^{-1}\right)$, where $\rho(M)$ represents the spectral radius of Matrix $M, F$ is the rate of occurring of new infections, and $V$ is the rate of transferring individuals outside the original group. Therefore, the basic reproduction number $R_{E}$ of model system (1) is

$$
R_{E}=\frac{\beta \Lambda}{\mu(\gamma+\sigma+\mu)}\left\{\frac{\mu+\alpha \varepsilon}{\mu+\varepsilon}\right\}
$$

In the absence of public health education, $\varepsilon=0$ and the basic reproduction number becomes

$$
R_{0}=\frac{\beta \Lambda}{\mu(\gamma+\sigma+\mu)}
$$

Using (16), we can express (15) as

$$
R_{E}=R_{0}\left\{\frac{\mu+\alpha \varepsilon}{\mu+\varepsilon}\right\}
$$

Since we have set $0<\varepsilon<1$, it follows that $\left\{\frac{\mu+\alpha \varepsilon}{\mu+\varepsilon}\right\}<1$, which implies that $R_{E}<R_{0}$. When $\varepsilon=0$, we have $R_{E}=R_{0}$. Thus, we see from expression (17) that public health educational interventions have a positive impact on the reduction of new infections.

It is important to note that we have used the next-generation approach in estimating the basic reproduction number; however, there exist other methods, including obtaining the eigenvalues of the Jacobian matrix, the survival function technique, and the existence of the endemic equilibrium, which can be employed in models where calculations are unsuccessful [35].

\subsection{Local stability of the disease-free equilibrium}

The disease-free equilibrium points of the model are its steady state solutions in the absence of infection or disease. Again following Theorem 2 in Van den Driessche and Watmough [34], we present local stability property for the disease-free equilibrium in the following theorem.

Theorem 3 The disease-free equilibrium of model system (1) is locally asymptotically stable whenever $R_{E}<1$ and unstable if $R_{E}>1$. 
Proof We prove Theorem 3 by obtaining the eigenvalues of the Jacobian matrix and show that they have negative real parts.

The Jacobian matrix of model system (1) is given by

$$
J=\left(\begin{array}{ccc}
-\beta I-(\varepsilon+\mu) & -\beta S+\gamma & 0 \\
\beta I & \beta S+\alpha \beta E-(\gamma+\sigma+\mu) & \alpha \beta I \\
\varepsilon & -\alpha \beta E & -(\alpha \beta I+\mu)
\end{array}\right) .
$$

The Jacobian matrix at DFE, $\left(S^{0}, E^{0}, I^{0}\right)$ is given by

$$
J\left(D^{0}\right)=\left(\begin{array}{ccc}
-(\varepsilon+\mu) & \gamma-\left(\frac{\beta \Lambda}{\varepsilon+\mu}\right) & 0 \\
0 & \left(\frac{\beta \Lambda}{\varepsilon+\mu}\right)+\left(\frac{\alpha \beta \varepsilon \Lambda}{\mu(\varepsilon+\mu)}\right)-(\gamma+\sigma+\mu) & 0 \\
\varepsilon & -\left(\frac{\alpha \beta \varepsilon \Lambda}{\mu(\varepsilon+\mu)}\right) & -\mu
\end{array}\right) .
$$

The characteristic equation is given by

$$
\left|J\left(D^{0}\right)-\lambda I\right|=0
$$

We compute eigenvalues of the equation

$$
\left|J\left(D^{0}\right)-\lambda I\right|=\left|\begin{array}{ccc}
-(\varepsilon+\mu) & \gamma-\left(\frac{\beta \Lambda}{\varepsilon+\mu}\right) & 0 \\
0 & \frac{\beta \mu \Lambda+\alpha \beta \Lambda \varepsilon-\mu(\varepsilon+\mu)(\gamma+\sigma+\mu)}{\mu(\varepsilon+\mu)}-\lambda & 0 \\
\varepsilon & 0 & -(\mu+\lambda)
\end{array}\right|=0 .
$$

The eigenvalues are obtained as follows:

$$
\lambda_{1}=-(\varepsilon+\mu), \lambda_{2}=-\mu \text { and } \lambda_{3}=\left(\frac{\beta \mu \Lambda+\alpha \beta \Lambda \varepsilon-\mu(\varepsilon+\mu)(\gamma+\sigma+\mu)}{\mu(\varepsilon+\mu)}\right)=(\gamma+\sigma+\mu)\left(R_{E}-1\right) \text {. If } R_{E}<1 \text {, }
$$
then $\lambda_{1}, \lambda_{2}$, and $\lambda_{3}$ are all negative. The eigenvalues have negative real parts. It follows that the trace of $J\left(D^{0}\right)$ is negative and the determinant is positive. Therefore the disease-free equilibrium (DFE) is locally asymptotically stable in the region $\Gamma$ if and only if $R_{E}<1$. Thus, Theorem 3 is proved.

\subsection{Global stability of the disease-free equilibrium}

Theorem 4 The disease-free equilibrium $\left(D^{0}\right)$ of model system (1) is globally asymptotically stable whenever $R_{E}<1$ and unstable if $R_{E}>1$

Proof We use geometry to prove the global stability of the disease-free equilibrium (DFE) of model system (1). From Theorem 3, we know that if $0<R_{E}<0$, the equilibrium $D^{0}$ is locally asymptotically stable and there are no endemic equilibria in the region $\Gamma$ when $0<R_{E}<1$. According to Perko [36], any solution of model system (1) starting in $\Gamma$ must approach either an equilibrium or a closed orbit in $\Gamma$. With reference to Kelley and Peterson [37], if the solution path approaches a closed orbit, then this closed orbit must enclose an equilibrium. Nevertheless, the equilibrium existing in $\Gamma$ is $D^{0}$ when $0<R_{E}<1$ and it is located in the boundary of $\Gamma$, therefore there is no closed orbit in $\Gamma$. Hence, any solution of system (1) with initial condition in $\Gamma$ must approach the point $D^{0}$ as $t \rightarrow \infty$. Therefore, the disease-free equilibrium $D^{0}$ is globally asymptotically stable in $\Gamma$ when $0<R_{E}<1$. When $R_{E}>1$, then $\lambda_{3}>0$, that is, the root will be positive. Therefore, the equilibrium $D^{0}$ will be unstable. Thus, we have proved Theorem 4 . 


\subsection{Existence of a unique positive endemic equilibrium $\varphi_{*}\left(S_{*}, E_{*}, I_{*}\right)$}

Theorem 5 An endemic equilibrium $\varphi_{*}\left(S_{*}, E_{*}, I_{*}\right)$ exists provided that $R_{E}>1$.

Proof At an endemic state, equation (1) becomes

$$
\begin{aligned}
& \Lambda-\beta S_{*} I_{*}-(\varepsilon+\mu) S_{*}+\gamma I_{*}=0, \\
& \varepsilon S_{*}-\alpha \beta E_{*} I_{*}-\mu E_{*}=0, \\
& \beta S_{*} I_{*}+\alpha \beta E_{*} I_{*}-(\gamma+\sigma+\mu) I_{*}=0 .
\end{aligned}
$$

To calculate endemic equilibrium (EE), we set $\varphi_{*}\left(S_{*}, E_{*}, I_{*}\right) \neq 0$.

Solving for $S_{*}$ in equation (18), we obtain

$$
S_{*}=\frac{\Lambda+\gamma I_{*}}{\beta I_{*}+(\varepsilon+\mu)} .
$$

Solving for $E_{*}$ in equation (19), we get

$$
E_{*}=\frac{\varepsilon S_{*}}{\left(\alpha \beta I_{*}+\mu\right)} .
$$

We again solve for $S_{*}$ in equation (20) and obtain

$$
S_{*}=\frac{(\gamma+\sigma+\mu)-\alpha \beta E_{*}}{\beta} .
$$

Equating equation (21) to equation (23) and solving for $E_{*}$, we obtain

$$
E_{*}=\left\{\frac{\gamma+\sigma+\mu}{\alpha \beta}\right\}-\frac{1}{\alpha}\left\{\frac{\Lambda+\gamma I_{*}}{\beta I_{*}+(\varepsilon+\mu)}\right\} .
$$

Substituting (23) into (22) and simplifying, we have

$$
E_{*}=\frac{\frac{\varepsilon}{\beta}(\gamma+\sigma+\mu)}{\left(\alpha \beta I_{*}+\mu+\alpha \varepsilon\right)} .
$$

To solve for $I_{*}$, we equate equations (24) and (25) and simplify to get

$$
\begin{aligned}
0= & \alpha^{2} \beta^{2}(\sigma+\mu) I_{*}^{2}+\alpha \beta(\alpha \Lambda+\mu \gamma+2 \alpha \varepsilon \gamma+\alpha \varepsilon \sigma+\alpha \varepsilon \mu) \\
& -(\gamma+\sigma+\mu)\left(\alpha \beta \mu+2 \alpha^{2} \beta \varepsilon+\alpha^{2} \beta \mu\right) I_{*} \\
& +\alpha\left(\Lambda \mu \beta+\Lambda \alpha \beta \varepsilon+\alpha \varepsilon^{2} \gamma+\alpha \varepsilon \gamma \mu+\alpha \varepsilon^{2} \sigma+\alpha \varepsilon \sigma \mu+\alpha \varepsilon^{2} \mu+\alpha \varepsilon \mu^{2}\right) \\
& -\alpha(\gamma+\sigma+\mu)\left(\varepsilon \mu+\alpha \varepsilon^{2}+\mu^{2}+\alpha \varepsilon \mu\right)=0
\end{aligned}
$$

Equation (26) can be expressed as

$$
A I_{*}^{2}+B I_{*}+C=0
$$


where

$$
\begin{aligned}
A= & \alpha^{2} \beta^{2}(\sigma+\mu), \\
B= & \alpha \beta(\alpha \Lambda+\mu \gamma+2 \alpha \varepsilon \gamma+\alpha \varepsilon \sigma+\alpha \varepsilon \mu)-(\gamma+\sigma+\mu)\left(\alpha \beta \mu+2 \alpha^{2} \beta \varepsilon+\alpha^{2} \beta \mu\right), \\
C= & \alpha\left(\Lambda \mu \beta+\Lambda \alpha \beta \varepsilon+\alpha \varepsilon^{2} \gamma+\alpha \varepsilon \gamma \mu+\alpha \varepsilon^{2} \sigma+\alpha \varepsilon \sigma \mu+\alpha \varepsilon^{2} \mu+\alpha \varepsilon \mu^{2}\right) \\
& -\alpha(\gamma+\sigma+\mu)\left(\varepsilon \mu+\alpha \varepsilon^{2}+\mu^{2}+\alpha \varepsilon \mu\right) .
\end{aligned}
$$

To obtain $I_{*}$, we solve the quadratic equation (27) by determining the value of $C$ in expression (28) using

$$
I_{*}=\frac{-B \pm \sqrt{B^{2}-4 A C}}{2 A} .
$$

Since $R_{E}=\frac{\beta \Lambda}{\mu(\gamma+\sigma+\mu)} \frac{\mu+\alpha \varepsilon}{\mu+\varepsilon}>1$, it is easy to show from equation (28) that

$$
\begin{aligned}
& \alpha(\gamma+\sigma+\mu)\left(\varepsilon \mu+\alpha \varepsilon^{2}+\mu^{2}+\alpha \varepsilon \mu\right) \\
& \quad>\alpha\left(\Lambda \mu \beta+\Lambda \alpha \beta \varepsilon+\alpha \varepsilon^{2} \gamma+\alpha \varepsilon \gamma \mu+\alpha \varepsilon^{2} \sigma+\alpha \varepsilon \sigma \mu+\alpha \varepsilon^{2} \mu+\alpha \varepsilon \mu^{2}\right) .
\end{aligned}
$$

This proves that $C<0$, when $R_{E}>1$. Clearly, equation (27) can only be expressed as

$$
(A) I_{*}^{2}-(B) I_{*}-(C)=0
$$

The equation $I_{*}=0$ corresponds to a situation when there is no disease in the population. In case of backward bifurcation, multiple endemic equilibria must exist. This implies that while considering equation (26), there are three cases we have to consider depending on the signs of $B$ and $C$ since $A$ is always positive. That is,

(a) If $B<0$ and $C=0$ or $\sqrt{B^{2}-4 A C}=0$, then equation (26) has a unique disease-endemic equilibrium point (one positive root) and there is no possibility of backward bifurcation.

(b) If $C>0$ and $B>0$ and $B^{2}-4 A C>0$, then equation (26) has two endemic equilibria (two positive roots), and thus there is possibility of backward bifurcation to occur.

(c) Otherwise, there is none.

However, it is important to note that $C$ is always positive if $R_{E}<1$ and negative if $R_{E}>1$. Hence the above explanation leads to the following theorem.

\section{Theorem 6 The jigger infection model has}

(i) Precisely one unique endemic equilibrium if $C<0 \Leftrightarrow R_{E}>0$.

(ii) Precisely one unique endemic equilibrium if $B<0$ and $C=0$ or $B^{2}-4 A C=0$.

(iii) Precisely two unique endemic equilibria if $C>0$, and $B<0$ or $B^{2}-4 A C>0$.

(iv) None, otherwise.

From (29) it is observed that there is only one positive root of equation (27). That is, $I_{*}>0$. We can therefore conclude that there exists one unique positive disease-endemic equilibrium. 


\subsection{Local stability of the endemic equilibrium}

Theorem 7 If $R_{E}>1$, then the disease-endemic equilibrium of model system (1) is locally asymptotically stable.

Proof A disease is endemic in a population if it persists in the population. We investigate the stability of the endemic equilibrium using the trace and the determinant. The Jacobian matrix at $\varphi_{*}\left(S_{*}, E_{*}, I_{*}\right)$ is given by

$$
J\left(\varphi_{*}\right)=\left(\begin{array}{ccc}
-\beta I_{*}-(\varepsilon+\mu) & -\beta S_{*}+\gamma & 0 \\
\beta I_{*} & \beta S_{*}+\alpha \beta E_{*}-(\gamma+\sigma+\mu) & \alpha \beta I_{*} \\
\varepsilon & -\alpha \beta E_{*} & -\left(\alpha \beta I_{*}+\mu\right)
\end{array}\right) .
$$

We substitute the values of $\varphi_{*}\left(S_{*}, E_{*}, I_{*}\right)$, then simplify to obtain the trace $\tau\left(U_{\varphi_{*}}\right)$. It can be seen from the Jacobian matrix that the trace $(\tau)$ of $J_{\varphi_{*}}$ is negative and the determinant is positive. That is,

$$
\begin{aligned}
\operatorname{Det}\left(J_{\varphi_{*}}\right)= & \left|\begin{array}{ccc}
-\beta I_{*}-(\varepsilon+\mu) & -\beta S_{*}+\gamma & 0 \\
\beta I_{*} & -\left\{(\gamma+\sigma+\mu)-\beta S_{*}-\alpha \beta E_{*}\right\} & \alpha \beta I_{*} \\
\varepsilon & -\alpha \beta E_{*} & -\left(\alpha \beta I_{*}+\mu\right)
\end{array}\right|, \\
= & \left(\beta I_{*}\left(\alpha \beta I_{*}+\mu\right)+\alpha \varepsilon \beta I_{*}\right)\left(\gamma+\beta S_{*}\right) \\
& +\frac{\mu\left(\alpha \beta I_{*}+\mu\right)(\gamma+\sigma+\mu)(\mu+\varepsilon)\left(S_{*}+\alpha D_{*}\right)}{\Lambda\left(\alpha^{2} \beta^{2} I_{*} D_{*}+(\gamma+\sigma+\mu)\right)(\mu+\alpha \varepsilon)}\left[R_{E}-1\right] .
\end{aligned}
$$

Clearly, $\operatorname{Det}\left(J_{\varphi_{*}}\right)>0$ if and only if $R_{E}>1$.

Therefore, we can conclude that model system (1) has an asymptotically stable endemic equilibrium whenever $R_{E}>1$. Thus, Theorem 7 is proved.

\subsection{Global stability of the endemic equilibrium}

We study the global asymptotic stability of the endemic equilibrium using the Lyapunov direct method and LaSalle's invariance principle $[38,39]$.

Theorem 8 If $R_{E}>1$, then the endemic equilibrium of model system (1) is globally asymptotically stable.

Proof To prove the theorem, we begin by constructing a suitable Lyapunov linear and quadratic function of the form

$$
L=\Sigma_{i=1}^{3} U_{i}\left(x_{i}-x_{i}^{*} \ln x_{i}\right)
$$

where $U_{i}$ is a properly selected constant, $x_{i}$ is the population of $i$ th compartment, $x_{i}^{*}$ is the equilibrium value of $x_{i}$ and $U_{i}>0$. The Lyapunov function denoted by $L$ is continuous and differentiable. We have

$$
L=\Sigma_{i=3}^{3} U_{i}\left(x_{i}-x_{i}^{*} \ln x_{i}\right)
$$


The global stability of the endemic equilibrium holds if its time derivative $\frac{d L}{d t} \leq 0$. The time derivative of the Lyapunov function $L$ is given by

$$
\begin{aligned}
\frac{d L}{d t}= & U_{1}\left(1-\frac{S^{*}}{S}\right) \frac{d S}{d t}+U_{2}\left(1-\frac{I^{*}}{I}\right) \frac{d I}{d t}+U_{3}\left(1-\frac{E^{*}}{E}\right) \frac{d E}{d t} \\
\frac{d L}{d t}= & -U_{1}\left(1-\frac{S^{*}}{S}\right)^{2}(\epsilon+\mu) S+U_{1}\left(1-\frac{S^{*}}{S}\right)\left(\frac{I^{*}}{I} \frac{S^{*}}{S}-1\right) \beta S I \\
& -U_{2}\left(1-\frac{E^{*}}{E}\right)^{2} \mu E+U_{2}\left(1-\frac{E^{*}}{E}\right)\left(\frac{E^{*}}{E} \frac{I^{*}}{I}-1\right) \alpha \beta E I \\
& -U_{3}\left(1-\frac{I^{*}}{I}\right)^{2}(\gamma+\sigma+\mu) I .
\end{aligned}
$$

We adopt the approach used in [40] and have the following expression:

$$
\begin{aligned}
\frac{d L}{d t}= & -U_{1}\left(1-\frac{S^{*}}{S}\right)^{2}(\epsilon+\mu) S-U_{2}\left(1-\frac{E^{*}}{E}\right)^{2} \mu E \\
& -U_{3}\left(1-\frac{I^{*}}{I}\right)^{2}(\gamma+\sigma+\mu) I+Z\left(\varphi_{*}\right),
\end{aligned}
$$

where

$$
Z\left(\varphi_{*}\right)=U_{1}\left(1-\frac{S^{*}}{S}\right)\left(\frac{I^{*}}{I} \frac{S^{*}}{S}-1\right) \beta S I+U_{2}\left(1-\frac{E^{*}}{E}\right)\left(\frac{E^{*}}{E} \frac{I^{*}}{I}-1\right) \alpha \beta E I \leq 0 .
$$

$Z\left(\varphi_{*}\right)$ is non-positive by following the approach of Korobeinikov [29] and McCluskey [40]. Thus, $Z\left(\varphi_{*}\right) \leq 0$ for all $\varphi \geq 0$. Hence, $d L / d t \leq 0$ in $\varphi$ and is zero when $\varphi=\varphi_{*}$. Therefore, the largest invariant set in $\varphi$ such that $d L / d t=0$ is the singleton $\left(\varphi_{*}\right)$ which is our endemic equilibrium point. By LaSalle's invariant principle [39] we conclude that $\left(\varphi_{*}\right)$ is globally asymptotically stable. This completes the proof of Theorem 8.

\section{Numerical simulations}

In order to illustrate our theoretical results, we carry out numerical simulations for the mathematical model using the set of parameters from literature as shown in Table 1 . The values of some parameters in our model are assumed.

\section{Discussion}

Figure 2 shows the local stability of disease-free equilibrium when $R_{E}=0.19090<1$ as $t \rightarrow \infty$ for susceptible (a), educated (b), and jigger-infested (c) populations. From the figure, we note that the number of susceptible individuals (see Fig. 2(a)) is more than the

Table 1 Parameter values used in Figs. 2, 3, 4, 5 and 6

\begin{tabular}{llll}
\hline Parameter & Symbol & Value & Source \\
\hline Recruitment rate of susceptible class & $\Lambda$ & $4.4 \times 10^{-3} \mathrm{day}^{-1}$ & {$[41]$} \\
Effective contact rate & $\beta$ & $1.4989 \times 10^{-2} \mathrm{day}^{-1}$ & {$[42]$} \\
Dissemination rate & $\varepsilon$ & $1.431 \times 10^{-2} \mathrm{day}^{-1}$ & Assumed \\
Scaling factor & $\alpha$ & $0.2-0.990$ & Assumed \\
Disease-induced mortality rate & $\sigma$ & $5.0 \times 10^{-2} \mathrm{day}^{-1}$ & {$[42]$} \\
Recovery rate & $\gamma$ & $4.27 \times 10^{-1} \mathrm{day}^{-1}$ & {$[42]$} \\
Per capita natural death rate & $\mu$ & $1.6 \times 10^{-2}$ day $^{-1}$ & {$[41]$} \\
\hline
\end{tabular}




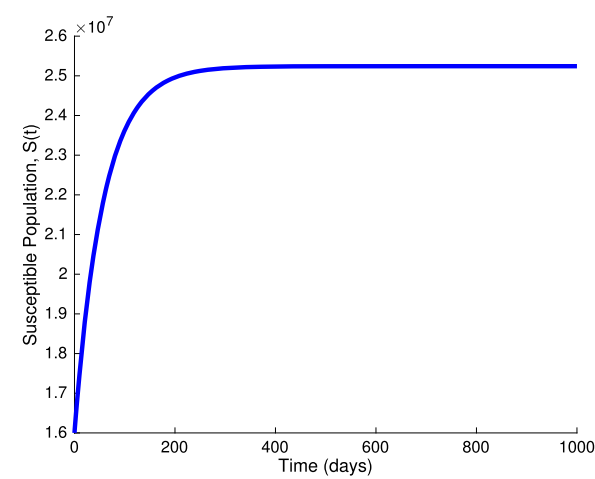

(a)

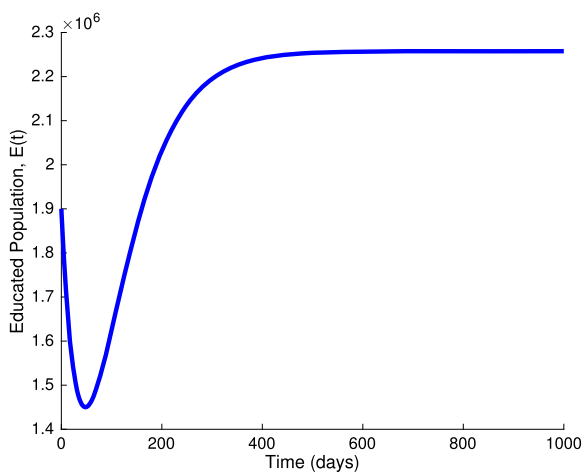

(b)

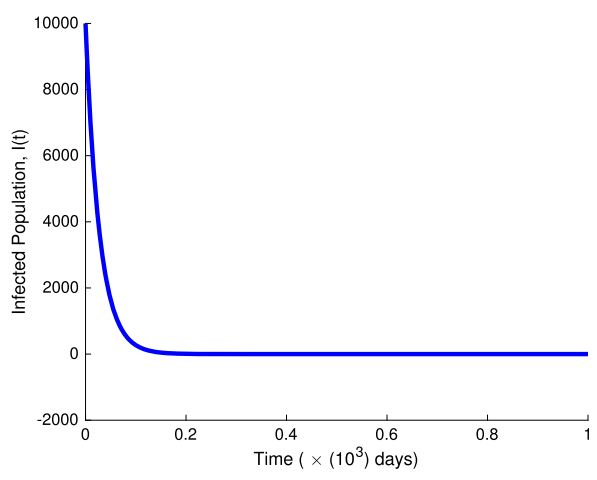

(c)

Figure 2 The dynamics of susceptible (a), educated (b), and infected (c) populations in model system (1) when $R_{E}=0.19090<1$. Used parameter values are in Table 1

number of educated individuals (see Fig. 2(b)). This may be due to the fact that public health education is not necessary at this time since there are still no infections in the population. In Fig. 2(c) we also observe that the number of jigger-infested individuals drops rapidly to zero as the number of educated individuals increases. This confirms our theoretical findings that public health education is a very effective control measure in eradicating the jigger menace.

Figure 3 shows that the disease-endemic equilibrium $\varphi_{*}$ is locally asymptotically stable when $R_{E}=1.231>1$ as $t \rightarrow \infty$ for the susceptible and educated populations (a) and the jigger-infested population (b). Figure 3(b) depicts a sharp increase in the number of infested individuals at the onset of an infection before the stability status is attained. This could be attributed to the decrease in the educated population in Fig. 3(a) which has consequently resulted in an increase in the number of susceptible individuals. This implies that if the control measures are implemented whenever there is an outbreak of jigger infestation, then the disease prevalence is most likely to be contained.

In Fig. 4(a), (b), and (c), we present the time evolution of the density of susceptible, educated, and jigger-infested populations, respectively, with different initial values when $R_{E}=0.19090<1$. From Fig. 4, we note that a steady increase in the number of educated population leads to a very low number of susceptible population, which consequently results in a decrease in the number of infected individuals, and vice versa. We also observe that jigger menace can be effectively eliminated from the community after a given period 


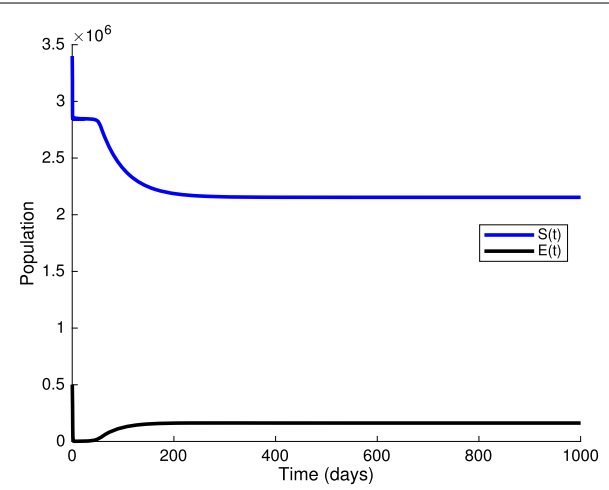

(a)

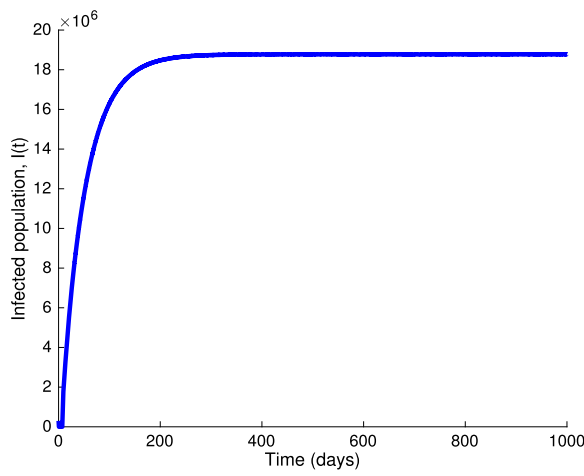

(b)

Figure 3 The dynamics of model system (1) when $R_{E}=1.231>1$ for the susceptible and educated populations (a) and the infected population (b) based on parameter values in Table 1

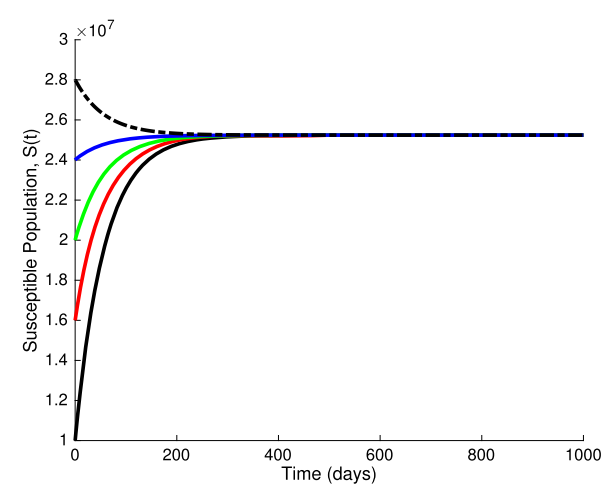

(a)

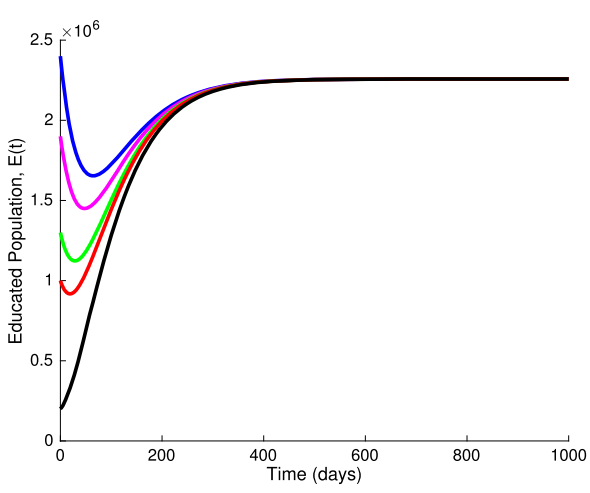

(b)

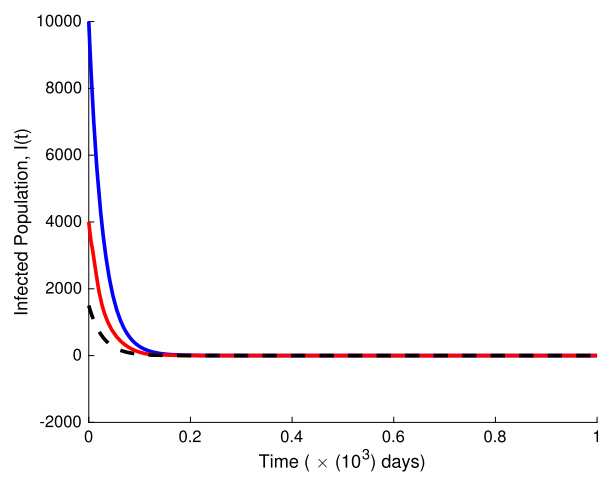

(c)

Figure 4 Global stability of the disease-free equilibrium. (a): global stability of the disease-free equilibrium when $R_{E}=0.19090<1$ for the susceptible population. (b): global stability of the disease-free equilibrium when $R_{E}=0.19090<1$ for the educated population. (c): global stability of the disease-free equilibrium when $R_{E}=0.19090<1$ for the infected population. Parameter values are available in Table 1

of time through public health education regardless of the number of individuals who are initially infested. Theorems 3 and 4 are numerically illustrated in Fig. 4. 


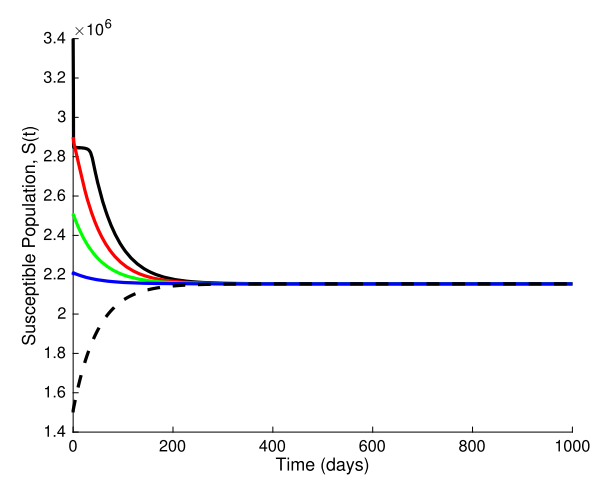

(a)

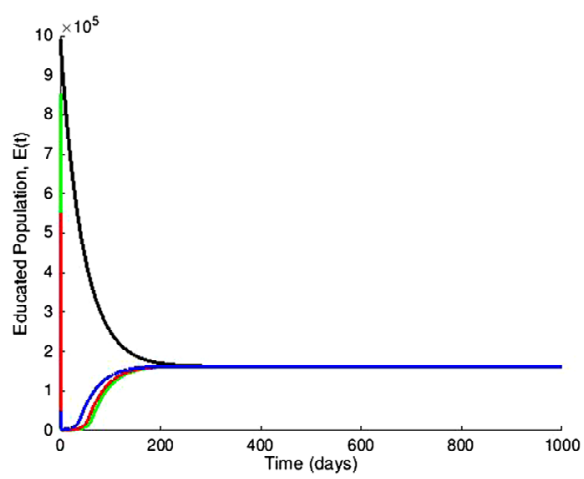

(b)

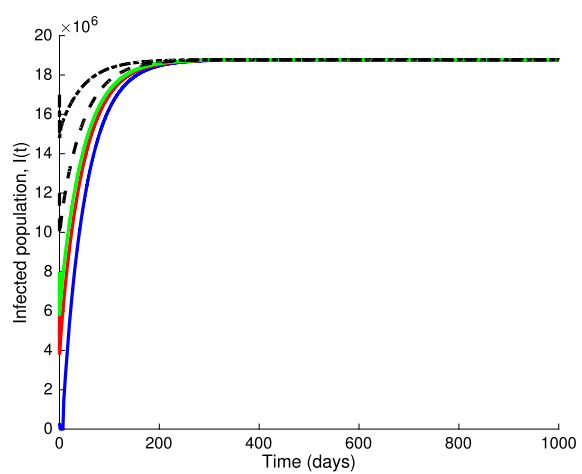

(c)

Figure 5 Global stability of the disease-endemic equilibrium when $R_{E}>1$. (a): global stability of the disease-endemic equilibrium for the susceptible population. (b): global stability of the disease-endemic equilibrium for the educated population. (c): global stability of the disease-endemic equilibrium for the jigger-infested population. The used parameter values are available in Table 1

In Fig. 5(a), (b), and (c), we present the time evolution of the density of susceptible, educated, and jigger-infested populations, respectively with different initial values when $R_{E}=1.231$, which is greater than unity. The numerical result illustrated in Fig. 5 confirms that model system (1) has only one unique positive endemic equilibrium point when $R_{E}>1$. This implies that there is no bifurcation and the disease will persist in the population if control measures are not put in place whenever there is an outbreak.

Finally, in Fig. 6(a), we study the influence of parameter $\alpha$ on the total density of infested individuals with $\alpha=0.2,0.4,0.6$ when $R_{E}=0.7671<1$, where we establish that decreasing the value of $\alpha$ could accelerate the extinction of the jigger menace. Figure 6(b) depicts the effect of different values of the parameter $\alpha$ with $R_{E}=1.2783>1$ on the total density of jigger-infested individuals. From Fig. 6, we observe that when $\alpha$ approaches one, the disease approaches an endemic equilibrium point. That is, the infection persists in the population. This implies that when $R_{E}>1$, public health education does not completely change the value of the epidemic threshold $R_{0}$, but it reduces the final density of the number of jigger-infested individuals. From this observation, we recommend that measures that can reduce $R_{0}$ be undertaken to help fight the disease. This is a confirmation that effective public health education is necessary in controlling jigger infestation. This also supports our theoretical analysis where we showed that public health education effectively reduces the basic reproduction number of the model. 


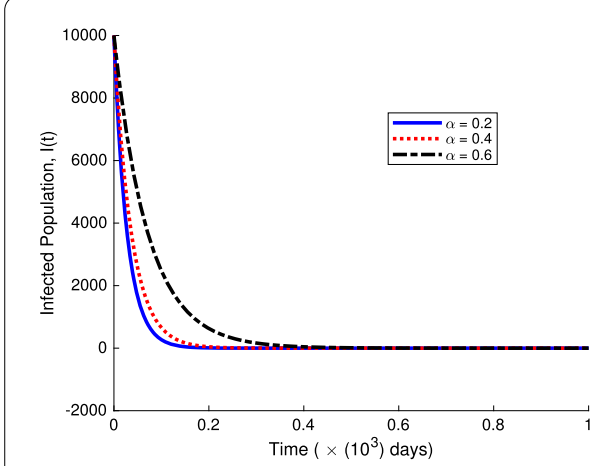

(a)

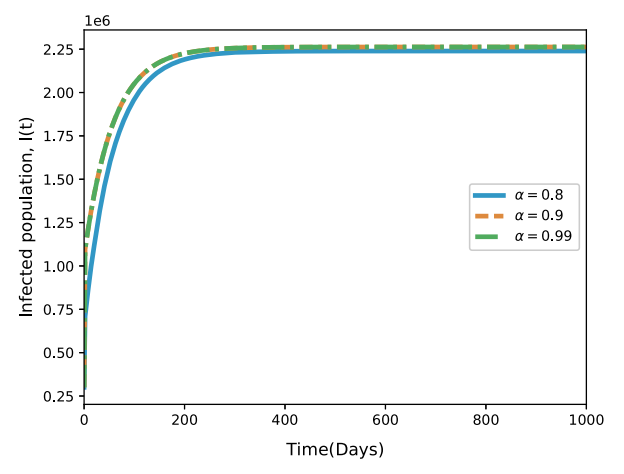

(b)

Figure 6 The influence of different values of $\alpha$ on the total density of jigger infested individuals. For (a), $\alpha=0.2,0.4,0.6$ with $R_{E}=0.7671$, whereas $\alpha=0.80,0.90,0.99$ for $(\mathbf{b})$ with $R_{E}=1.2783$. Other parameter values are as displayed in Table 1

\section{Conclusion}

In this paper, the jigger infestation mathematical model with public health education as an intervention strategy was formulated and comprehensively analyzed. Using the theory of differential equations, the invariant set in which the solutions of the model are biologically meaningful was derived. Boundedness of solutions was also proved. Analysis of the model showed that there exist two possible solutions, namely the disease-free equilibrium point and the positive endemic equilibrium point. Further analysis showed that both the diseasefree and the endemic equilibria points are locally and globally asymptotically stable. It means that the jigger menace will disappear if $R_{E}<1$, otherwise, it will be prevalent if $R_{E}>1$.

Numerical simulations have been performed to support the analytical results. At the same time, we have discussed the influence of different values of $\alpha$ on the total density of the jigger-infested individuals, establishing that public health education is an effective measure of controlling the jigger menace as it reduces the spreading threshold. From this work, we recommend that control measures be put in place especially in resource-poor communities where the disease is endemic. Even though tungiasis is a public health hazard, reliable data on the disease occurrence are very scarce. The disease is in many ways a hidden disease that is generally neglected by healthcare providers and political decision makers.

This work provides a basic dynamical model that can be used to understand the impact of public health education on jigger infestation. Finally, the model has mass-action transmission, which assumes homogeneous mixing; however, in reality, the contact process of population cannot be uniform collision, different people contact person may be entirely different per unit of time. For future work, we propose that complex networks [31, 32, 43-46], which consider the mechanisms of epidemic transmission in large-scale social networks with distinct heterogeneities, be incorporated into the model. 
Funding

This work was partially supported by China Scholarship Council under CSC No. 2015404006 and Taita Taveta University.

\section{Competing interests}

The authors declare that they have no competing interests.

\section{Authors' contributions}

RAN designed the study, performed the mathematical analysis of the model and discussions, and also wrote and typeset the manuscript. DNA and TOO performed numerical simulations. TOO also edited the manuscript. CMM proofread the final manuscript. All authors read and approved the final manuscript.

\section{Author details}

'Department of Mathematics, Shanghai University, Shanghai, China. ${ }^{2}$ Department of Mathematics and Informatics, Taita Taveta University, Voi, Kenya. ${ }^{3}$ Department of Mathematics, Masinde Muliro University of Science and Technology, Kakamega, Kenya. ${ }^{4}$ Institute of Mathematical Sciences, Strathmore University, Nairobi, Kenya.

\section{Publisher's Note}

Springer Nature remains neutral with regard to jurisdictional claims in published maps and institutional affiliations.

Received: 8 May 2018 Accepted: 8 November 2018 Published online: 20 November 2018

\section{References}

1. Veraldi, S., Valsecchi, M.: Imported tungiasis: a report of 19 cases and review of the literature. Int. J. Dermatol. 46(10), 1061-1066 (2007)

2. Kamau, T.M., Ngechu, R.N., Haile, Z.T., Mwitari, J.: An exploration of factors associated with jigger infestation (tungiasis) among residents of Muranga North District, Kenya. Int. J. Health Sci. Res. 4(3), 1-8 (2014)

3. Ehrenberg, J.P., Ault, S.K.: Neglected diseases of neglected populations: thinking to reshape the determinants of health in Latin America and the Caribbean. BMC Public Health 5(1), 119 (2005)

4. Heukelbach, J., De Oliveira, F.A.S., Hesse, G., Feldmeier, H.: Tungiasis: a neglected health problem of poor communities. Trop. Med. Int. Health 6(4), 267-272 (2001)

5. Heukelbach, J., Wilcke, T., Harms, G., Feldmeier, H.: Seasonal variation of tungiasis in an endemic community. Am. J. Trop. Med. Hyg. 72(2), 145-149 (2005)

6. AKT: The Jigger menace in Kenya. Anti-jigger magazine. Ahadi Kenya Trust (October 2018 (2018)). Available at from http://www.jigger-ahadi.org/Anti

7. MPHS: Kenya National Assembly Official Record (Hansard). The parliamentary ministerial statement on tungiasis in Kenya. Ministry of Public Health and Sanitation. Available at from

http://www.parliament.go.ke/the-national-assembly/offices/hansard-department. (Retrieved October 2018), (2009)

8. Ngomi, N.N.: Tungiasis in 5-12 year olds and associated factors in Murang'a South district, Central Kenya. Abstracts of postgraduate thesis (2012)

9. Ruttoh, S.K., Ochieng'Omondi, D., Wanyama, N.I.: Tunga penetrans - a silent setback to development in Kenya. J. Environ. Sci. Eng. B 1(4B), 527-534 (2012)

10. Ugbomoiko, U.S., Ariza, L., Ofoezie, I.E., Heukelbach, J.: Risk factors for tungiasis in Nigeria: identification of targets for effective intervention. PLoS Negl. Trop. Dis. 1(3), 87 (2007)

11. Eisele, M., Heukelbach, J., Van Marck, E., Mehlhorn, H., Meckes, O., Franck, S., Feldmeier, H.: Investigations on the biology, epidemiology, pathology and control of tunga penetrans in Brazil: I. Natural history of tungiasis in man. Parasitol. Res. 90(2), 87-99 (2003)

12. Feldmeier, H., Eisele, M., Sabóia-Moura, R.C., Heukelbach, J.: Severe tungiasis in underprivileged communities: case series from Brazil. Emerg. Infect. Dis. 9(8), 949 (2003)

13. Feldmeier, H., Sentongo, E., Krantz, I.: Tungiasis (sand flea disease): a parasitic disease with particular challenges for public health. Eur. J. Clin. Microbiol. Infect. Dis. 32(1), 19-26 (2013)

14. Mwangi, J.N., Ozwara, H.S., Gicheru, M.M.: Epidemiology of tunga penetrans infestation in selected areas in Kiharu constituency, Murang'a County, Kenya. Trop. Dis. Travel Med. Vaccines 1(1), 13 (2015)

15. Yao, H., Liang, R.: Vaccination strategies of a modified SIS model on complex networks. IJCTT 4(3), 346-351 (2015)

16. Tchuenche, J.M., Dube, N., Bhunu, C.P., Smith, R.J., Bauch, C.T.: The impact of media coverage on the transmission dynamics of human influenza. BMC Public Health 11(1), 5 (2011)

17. Liu, R., Wu, J., Zhu, H.: Media/psychological impact on multiple outbreaks of emerging infectious diseases. Comput. Math. Methods Med. 8(3), 153-164 (2007)

18. Collinson, S., Heffernan, J.M.: Modelling the effects of media during an influenza epidemic. BMC Public Health 14(1), $376(2014)$

19. Huo, H.-F., Wang, Y.-Y.: Impact of media coverage on the drinking dynamics in the scale-free network. SpringerPlus 5(1), $204(2016)$

20. Huo, H.-F., Wang, Y.-Y.: Impact of media coverage on the drinking dynamics in the scale-free network. SpringerPlus 5(1), 204 (2016)

21. Mukandavire, Z., Garira, W., Tchuenche, J.: Modelling effects of public health educational campaigns on HIV/AIDS transmission dynamics. Appl. Math. Model. 33(4), 2084-2095 (2009)

22. Bhunu, C.P., Mushayabasa, S., Kojouharov, H., Tchuenche, J.: Mathematical analysis of an HIV/AIDS model: impact of educational programs and abstinence in sub-Saharan Africa. J. Math. Model. Algorithms 10(1), 31-55 (2011)

23. Feldmeier, H., Sentongo, E., Krantz, I.: Tungiasis (sand flea disease): a parasitic disease with particular challenges for public health. Eur. J. Clin. Microbiol. Infect. Dis. 32(1), 19-26 (2013)

24. Kahuru, J., Luboobi, L., Nkansah-Gyekye, Y.: Modelling the dynamics of Tungiasis transmission in zoonotic areas. Int. J. Comput. Math. Sci. 7(2), 375-399 (2017) 
25. Kahuru, J., Luboobi, L., Nkansah-Gyekye, Y.: Stability analysis of the dynamics of tungiasis transmission in endemic areas. Asian J. Math. Appl. 2017, Article ID ama0385 (2017)

26. Kahuru, J., Luboobi, L.S., Nkansah-Gyekye, Y: Optimal control techniques on a mathematical model for the dynamics of tungiasis in a community. International Journal of Mathematics and Mathematical Sciences 2017 (2017)

27. Nthiiri, K.J.: Mathematical modelling of jigger infection incorporating treatment as a control strategy. Int. Electron. J. Pure Appl. Math. 11(2), 87-97 (2017)

28. Diekmann, O., Heesterbeek, J.A.P., Metz, J.A.: On the definition and the computation of the basic reproduction ratio R0 in models for infectious diseases in heterogeneous populations. J. Math. Biol. 28(4), 365-382 (1990)

29. Korobeinikov, A., Wake, G.C.: Lyapunov functions and global stability for SIR, SIRS, and SIS epidemiological models. Appl. Math. Lett. 15(8), 955-960 (2002)

30. Anderson, R.M., May, R.M.: Infectious Diseases of Humans: Dynamics and Control. Oxford University Press, London (1992)

31. Pastor-Satorras, R., Castellano, C., Van Mieghem, P., Vespignani, A.: Epidemic processes in complex networks. Rev. Mod. Phys. 87(3), 925 (2015)

32. Pastor-Satorras, R., Vespignani, A.: Epidemic spreading in scale-free networks. Phys. Rev. Lett. 86(14), 3200 (2001)

33. Omondi, O.L., Wang, C., Xue, X., Lawi, O.G.: Modeling the effects of vaccination on rotavirus infection. Adv. Differ. Equ. 2015(1), $381(2015)$

34. Van den Driessche, P., Watmough, J.: Reproduction numbers and sub-threshold endemic equilibria for compartmental models of disease transmission. Math. Biosci. 180(1-2), 29-48 (2002)

35. Li, J., Blakeley, D., Smith, R.J.: The failure of RO. Comput. Math. Methods Med. (2011). https://doi.org/10.1155/2011/527610

36. Perko, L:: Differential Equations and Dynamical Systems, vol. 7. Springer, Berlin (2013)

37. Kelley, W.G., Peterson, A.C.: The Theory of Differential Equations: Classical and Qualitative. Springer, Berlin (2010)

38. La Salle, J., Lefschetz, S.: Stability by Liapunov's Direct Method with Applications, vol. 4. Elsevier, Amsterdam (2012)

39. LaSalle, J.P.: The Stability of Dynamical Systems, vol. 25. SIAM, Philadelphia (1976)

40. McCluskey, C.C.: Lyapunov functions for tuberculosis models with fast and slow progression. Math. Biosci. Eng. 3, 603-614 (2006)

41. CIA: The CIA World Factbook. CI Agency. Skyhorse Publishing, New York (2011)

42. KNBS: Kenya Demographic Profile. Available at https://reliefweb.int/sites/reliefweb.int/files/resources/Kenya. (Retrieved October 2018), (2014)

43. Barrat, A., Colizza, V., Vespignani, A.: Epidemic spreading and complex networks. Complex Networks 254 (2010)

44. Barabasi, A.-L., Albert, R.: Emergence of scaling in random networks. Science 286(5439), 509-512 (1999)

45. Pastor-Satorras, R., Castellano, C., Van Mieghem, P., Vespignani, A.: Epidemic processes in complex networks. Rev. Mod. Phys. 87(3), 925 (2015)

46. Keeling, M.J., Eames, K.T.: Networks and epidemic models. J. R. Soc. Interface 2(4), 295-307 (2005)

\section{Submit your manuscript to a SpringerOpen ${ }^{\circ}$ journal and benefit from:}

- Convenient online submission

- Rigorous peer review

- Open access: articles freely available online

- High visibility within the field

- Retaining the copyright to your article

Submit your next manuscript at $\boldsymbol{\nabla}$ springeropen.com 